\title{
A Commentary on 'Acute Non-traumatic Spinal Subdural Hematomas' Hénaux $\mathrm{PL}^{*}$ and Morandi $X$ \\ Department of Neurosurgery, Rennes University Hospital, Pontchaillou University Hospital, CHU Pontchaillou, Rennes Cedex 9, France
}

Acute non-traumatic spinal subdural hematomas (SSDH) are a rare occurrence among spinal hematomas [1]. They represent the least common form of extra-axial spinal hematomas (4.1\%) after epidural hematomas (75\%) and subarachnoid hematomas (15.7\%) [2]. They are mostly related to coagulopathy or anticoagulant therapy. Population ageing and growing anticoagulant administration, especially with the advent of the new ones [3], are likely to induce an increase in numbers of that pathology. Spinal hematoma typical clinical signs include an acute onset of a strong back knife-like pain at the level of the hemorrhage, then possibly followed by a pain-free interval (time interval between minutes to days). Thereafter progressive worsening of the neurological status (motor, sensory deficits and urinary disorders) may appear below the initial level of hemorrhage [2]. Even if no specific clinical sign can help physician to distinguish a SSDH from the other forms of spinal hematomas [2], SSDH pain-free interval (average time: $72 \mathrm{~h}$ ) is longer than epidural hematoma neurological deficits development (average time: $16 \mathrm{~h}$ ) [4]. Magnetic resonance imaging (MRI) is the dedicated imaging modality to make the diagnosis. Out of the context of coagulopathy or anticoagulant therapy, MRI with enhancement helps the physician check to rule out tumor [5] or vascular malformation possibly explaining why that hemorrhage occurred [6]. Today, we can even more identify a "spinal spot sign" [7], i.e., a contrast extravasation on MRI by analogy with those observed in hemorrhagic strokes. SSDH are usually located anterior and/or lateral around the spinal cord with a semi-circular shape, whereas epidural hematomas are rather located posterior to the spinal cord and looks like a lens on MRI [6]. Furthermore, their anatomic location in the subdural space allows them to spread over many levels of the spine. Thus, they can potentially threaten life if they reach cervical spine and in particular respiratory neural centers. Physician's decision making is influenced by age of the patient, his/her medical condition and clinical status. While it seems inconceivable not suggesting a surgery for a patient with significant neurological deterioration (excepting patients with poor medical conditions), there seems to be room for conservative management [8] in case of mild neurological impairment. Imaging data can help decision making. For example, "spinal spot sign" is a warning sign indicating a possible worsening of neurological clinical status due to possible expansion of the hematoma [7]. This type of information can convince the surgeon to operate on even in cases with mild neurological impairment. Mortality rate is $1.3 \%$ and morbidity rate $28 \%$ [9]. More generally, concerning spinal hematoma, prognosis is better when painfree interval is shorter, neurological deficits milder, delay to surgery shorter [2]. Absence of coagulopathy seems to lead to a better prognosis for SSDH [9]. No correlation seems to appear between prognosis and extent of the hemorrhage. However, in case of severe neurological deficits, surgery leads to a better clinical outcome than conservative management [2].

\section{References}

1. Morandi X, Riffaud L, Chabert E, Brassier G (2001) Acute non-traumatic spinal subdural hematomas in three patients. SPINE 26: E547-E551.

2. Kreppel D, Antoniadis G, Seeling W (2003) Spinal hematoma: A literature survey with meta-analysis of 613 patients. Neurosurg Rev 26: 1-49.

3. Castillo JM, Afanador HF, Manjarrez E, Morales XA (2015) Non-traumatic spontaneous spinal subdural hematoma in a patient with non-valvular atrial fibrillation during treatment with rivaroxaban. Am J Case Rep 16: 377-381.

4. Lao TT, Halpern SH, MacDonald D, Huh C (1993) Spinal subdural haematoma in a parturient after attempted epidural anaesthesia. Can $\mathrm{J}$ Anaesth $\mathrm{J}$ Can Anesth 40: 340-345.

5. Zhu YJ, Peng DQ, Shen F, Wang LL, Tang ZX, et al. (2015) Spontaneous thoracic ventral spinal subdural hematoma mimicking a tumoral lesion: A case report. J Med Case Rep 9: 132.

6. Payer M, Agosti R (2010) Spontaneous acute spinal subdural hematoma: Spontaneous recovery from severe paraparesis--Case report and review. Acta Neurochir (Wien) 152: 1981-1984.

7. Hauf M, Krestel H, Cavelti A, Biedermann AP, Schroth G, et al. (2015) Spinal spot sign. Neurologist 20: 104-105.

8. Oh SH, Han IB, Koo YH, Kim OJ (2009) Acute spinal subdural hematoma presenting with spontaneously resolving hemiplegia. J Korean Neurosurg Soc 45: 390-393.

9. Pereira BJA, de Almeida AN, Muio VMF, de Oliveira JG, de Holanda CVM, et al. (2016) Predictors of outcome in non-traumatic spontaneous acute spinal subdural hematoma: Case report and literature review. World Neurosurg 89: 574-577.

Received December 13, 2017; Accepted December 19, 2017; Published December 26, 2017

Citation: Hénaux PL, Morandi X (2017) A Commentary on 'Acute Non-traumatic Spinal Subdural Hematomas'. Int J Neurorehabilitation 4: 302. doi: 10.4172/23760281.1000302

Copyright: ( 2017 Hénaux PL, et al. This is an open-access article distributed under the terms of the Creative Commons Attribution License, which permits unrestricted use, distribution, and reproduction in any medium, provided the original author and source are credited. 